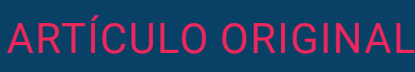

\title{
Programa de acompañamiento por pares para personas con diagnóstico reciente de infección por VIH: Experiencia PPP
}

\author{
Recibido: 12/8/20 Aceptado: 12/11/20
}

Edgardo G. Bottaro', Luciana Faraone², Agustina Soncini², Mónica Pasarón ${ }^{3}$, Nadia Roussilian², María Rosa Cascasi ${ }^{4}$, Álvaro Otreras ${ }^{1}$, Mara De Bernardi ${ }^{1}$, Sandra Rodríguez ${ }^{2}$, Sylvia Errea ${ }^{1}$, Daniela D'Alessandro ${ }^{1}$, Juliana Caccavo ${ }^{1}$, María Teresa Rodríguez Brieschke ${ }^{1}$, Beatriz Lauge ${ }^{1}$, María Magdalena Ilamendi', María del Carmen Espiño4, Pablo G. Scapellato'.

\section{RESUMEN}

Introducción: Para mejorar la retención en el sistema de salud de las personas que viven con VIH (PVVIH) con diagnóstico reciente y promover su adherencia se implementó el programa de acompañamiento de pares "Positivos para Positivos" (PPP).

Material y métodos: Se entrenó a PVVIH con excelente adherencia y se les ofreció integrar PPP. Entre 06/2014 y 08/2018 cada individuo con diagnóstico reciente de infección VIH fue invitado a contactar con PPP. Se evaluó prospectivamente la evolución de los pacientes durante un año y se analizaron variables vinculadas a adherencia. Se compararon sus resultados con lo observado entre PVVIH con diagnóstico reciente sin apoyo de pares. Se analizó mediante tablas de 2x2 y la prueba exacta de Fisher (Epilnfo7.2.2.6).

Resultados: Se incluyeron $158 \mathrm{PVVIH}$ (40 grupo intervención y 118 grupo control). En el grupo intervención hubo más pacientes que iniciaron TARGA [100\% vs $87,3 \%$; RR 1,15 (IC95 1,07-1,23); $p=0,024]$. Tras excluir a los derivados $y$ fallecidos tempranamente quedaron 37 y 112 pacientes respectivamente. En el grupo intervención se observó mejor control clínico [94,6\% vs 75,9\%; RR 4,2 (IC95 1,08$16,6) ; p=0,015]$ y menos abandono de seguimiento $[8,1 \%$ vs $25,9 \%$; RR 0,3 (IC95 0,11-0,98); $p=0,02$ ]. Entre quienes iniciaron TARGA y tuvieron al menos una consulta con el servicio de Infectología (37 grupo intervención y 97 grupo control) se registraron más pacientes con alta tasa de retiro de TARGA de farmacia [51,4\% vs 18,6\%; RR 2,77 (IC95 1,644,66); $p=0,0003]$; mayor alcance de $C V<50[100 \%$ vs $85,1 \%$; RR 1,18 (IC95 1,06-1,30); p=0,06]; y menos interrupciones

\begin{abstract}
${ }^{1}$ Servicio de Infectología, Hospital General de Agudos Donación F. Santojanni.

${ }^{2}$ Servicio Social, Hospital General de Agudos Donación F. Santojanni .

${ }^{3}$ Salud Mental, Hospital General de Agudos Donación F. Santojanni.

${ }^{4}$ Farmacia, Hospital General de Agudos Donación F. Santojanni.
\end{abstract}

Dirección para la correspondencia: Edgardo Bottaro, Pilar 950, CABA.

Email: egbottaro@gmail.com

No se recibió apoyo de ninguna fuente. Ninguno de los autores declara presentar conflictos de intereses en relación a esta publicación Los contenidos del material a ser evaluado no han sido publicados anteriormente.

no estructuradas del TARGA [10,8\% vs $36,1 \%$; RR 0,3 (IC95 $0,11-0,78) ; p=0,008]$

Conclusión: El acompañamiento de pares impactó positivamente en la adherencia de las PVVIH con diagnóstico reciente en el primer año de seguimiento.

Palabras clave: HIV, adherencia, pares, diagnóstico reciente. 


\section{Introducción}

El uso masivo del tratamiento antirretroviral de gran actividad (TARGA) para tratamiento de la infección por el virus de la inmunodeficiencia humana $(\mathrm{VIH})$ permitió alcanzar una notable reducción de la morbimortalidad por la infección (1), una significativa prolongación de la esperanza de vida de las personas que viven con VIH (PVVIH) (2), una profunda reducción de la transmisión vertical (3) y la casi eliminación de la transmisión horizontal $(4,5)$.

Para que el TARGA pueda ejercer su efecto beneficioso, las PVVIH deben mostrar una gran adherencia al mismo (6), pues la baja adherencia se asocia a supresión viral incompleta (7), desarrollo de resistencia viral (8), progresión de la enfermedady mayor mortalidad (9). Además, las variantes virales resistentes que emergen como resultado de la mala adherencia al TARGA podrán ser transmitidas a otras personas (10), lo que complejizará más aún el panorama epidemiológico, generando la aparición de cepas con resistencia primaria.

En 2004, la Organización Mundial de la Salud definió la adherencia terapéutica como "el grado en que el comportamiento de una persona -tomar el medicamento, seguir un régimen alimentario y ejecutar cambios del modo de vida- se corresponde con las recomendaciones acordadas con un prestador de asistencia sanitaria" (11). Siguiendo esta definición, la adecuada adherencia de los pacientes comprende la correcta toma de los fármacos (respetando dosis, intervalos, interacciones con otros fármacos, etc.), el cumplimiento de un calendario acordado de visitas médicas y la realización periódica de los análisis de laboratorio necesarios para la evaluación continua de la efectividad y seguridad, lo cual permitirá construir un escenario adecuado para la oportuna toma de decisiones. Todo eso requiere una estrecha colaboración entre el equipo de salud y el paciente.

El sistema sanitario reconoce la dificultad para mantener una buena adherencia por períodos prolongados $(12,13)$. Por ello es necesario identificar las barreras que atentan contra ella y desarrollar estrategias innovadoras adaptadas a cada situación (personal, geográfica, culturaly social) para sostenerlaymejorarla, ya que el mero traslado de experiencias exitosas de una parte del mundo a otra no garantiza que se replique el resultado.

Entre las múltiples estrategias existentes se halla la incorporación de pares: PVVIH que colaboran con el cuidado de otras PVVIH. La estrategia de asesoría de pares consiste en una intervención enfocada en la provisión de orientación y apoyo para la toma de decisiones informadas y racionales, provistas por una persona reconocida como un semejantea uno mismo (14). El objetivo es propiciar cambios a nivel individual, tratando de modificar los conocimientos, actitudes, creencias o comportamientos de la persona (15). La literatura refleja que los principales beneficios de esa estrategia consisten en reducir las conductas de riesgo para adquirir infecciones de transmisión sexual $(16,17)$, reducir la estigmatización y el temor a la misma $(18,19)$, contribuir a lograr mayor autonomía $(20,21)$ y a reaccionar mejor ante sentimientos vergonzantes (19) y mejorar el acceso y permanencia dentro del sistema de cuidados de la salud $(20,21)$.

En algunas experiencias los pares actuaron como modelos, compartiendo sus experiencias positivas con el consumo de TARGA (22). En otras, los pares son actores centrales en el cuidado de los pacientes, con buenos resultados (23, 24). Si bien en general las publicaciones muestran que el apoyo de pares beneficia la adherencia y la efectividad del tratamiento (25-29), otros autores comunican ausencia de beneficios $(30,31)$. Un metanálisis que incluyó 22 ensayos clínicos randomizados de distintas estrategias para promover la adherencia al TARGA mostró que la intervención de pares produjo una modesta mejoría de la adherencia solo cuando se combinó con otras intervenciones (32).

La mayoría de las experiencias de acompañamiento de pares en Latinoamérica focalizan en aspectos cualitativos (33-35), sin evaluar carga viral plasmática de $\mathrm{VIH}(\mathrm{CV})$, adherencia o recuento de CD4 (R-CD4).

Lo mismo sucede en Argentina. Un estudio descriptivo llevado a cabo en un hospital del conurbano bonaerense informa sobre un programa de apoyo entre pares diseñado para mejorar la adherencia de las PVVIH. Como resultante del mismo, al final del seguimiento la mayoría de los pacientes asistidos por pares consiguieron $\mathrm{CV}<400$ copias $\mathrm{ARN}-\mathrm{VIH} / \mathrm{mL}$. La falta de un grupo control impidió definir si la estrategia fue todo lo exitosa que los investigadores pretendían (36).

La población de PVVIH con diagnóstico reciente está poco estudiada en la literatura como blanco del apoyo de pares.

Un momento de particular labilidad emocional en la historia de las PVIH es el del diagnóstico (37), en el que el individuo pasa (en una concepción simplista pero así percibida por muchos sujetos) de sano a enfermo, aun cuando pueda no percibir ningún malestar físico. La vida se desestabiliza en un aspecto que hasta esa instancia no implicaba ninguna preocupación. Acontecen la tristeza, el temor y, en ocasiones, la desesperación. Se puede percibir a la muerte como una realidad inmediata y sobre estos 
sentimientos se pueden instalar la depresión y la ansiedad (38) con todas sus negativas repercusiones sobre la salud y sobre la futura e imprescindible adherencia (39).

Con la hipótesis de que el apoyo de pares podría influir positivamente en la adherencia de las PVVIH con diagnóstico reciente de infección por VIH, en el hospital General de Agudos D F Santojanni (HGADFS) se decidió desarrollar un programa para estimular su adherencia basado en la intervención de pares. Aquí se presentan los resultados tras 12 meses de ingreso al programa.

\section{Materiales y métodos}

A mediados de 2014 se empezó a trabajar en la elaboración de un programa para apoyar a $\mathrm{PVVIH}$ con diagnóstico reciente de infección por VIH, al que se denominó PPP, sigla de "Positivos para Positivos", nombre que juega con el doble significado de la palabra "positivo", pretendiendo que personas "VIHpositivas" puedan ser un acompañamiento "positivo" para otros individuos "VIH-positivos". Para eso se convocó a un equipo multidisciplinario compuesto por trabajadoras sociales, médicos, psicólogosy farmacéuticos que ya estaban trabajando en la temática dentro del hospital desde muchos años atrás.

La iniciativa se basó en la hipótesis de que una PVVIH con infección crónica por $\mathrm{VIH}$, y adherente al tratamiento, poseería herramientas útiles al momento de estimular y sostener la adherencia de PVVIH con diagnóstico reciente; herramientas desarrolladas a partir de "ya haber estado alli", lo que permitiría comprender y lograr mayor empatía con quien acaba de recibir su propio diagnóstico de infección por VIH.

Para la elección de los integrantes de PPP, los infectólogos pertenecientes al servicio de Infectología del HGADFS identificaron, entre las $\mathrm{PVVIH}$ que concurrían periódicamente al servicio para su propia atención, a quienes por sus características de excelente adherencia al TARGA, responsabilidad en el cuidado de la propia salud, buen estado clínico y $C V$ indetectable, podrían formar parte de un equipo de pares. El médico le ofrecía formar parte de PPP.

Quienes aceptaban la propuesta recibían una invitación formal por correo electrónico en la cual se explicaban los fundamentos del proyecto. Luego se concertaba una entrevista con alguna trabajadora social y/o la psicóloga integrantes del equipo de salud para evaluar su capacidad para formar parte de PPP. Quienes eran considerados aptos firmaban un consentimiento. Luego se llevaba a cabo una capacitación a cargo del equipo multidisciplinario en el que, a lo largo de cuatro encuentros presenciales de aproximadamente tres horas cada uno en las instalaciones del HGADFS, se instruía a los futuros integrantes de PPP sobre aspectos biológicos y farmacológicos de la infección por $\mathrm{VIH}$ y sobre aspectos vinculados a la relación con otras PVVIH. En reuniones previas, el equipo de salud diseñaba la dinámica a implementar en cada encuentro, el que era dirigido por uno de los profesionales de la salud idóneo en el tema a abordar. Durante cada encuentro se estimulaba a los participantes a intervenir y compartir propias vivencias y experiencias que enriquecieran a todos los participantes, generando un ámbito de intimidad y camaradería con la certeza de que el intercambio era nutritivo para todo el equipo.

La población blanco de la intervención era la de pacientes con diagnóstico reciente de infección por $\mathrm{VIH}$.

Se consideró que una persona tenía diagnóstico reciente de infección por VIH cuando la primera prueba serológica positiva tuviera una antigüedad igual o menor a seis meses respecto de la fecha de primera consulta al servicio de infectología del HGADFS.

Cada vez que una persona con diagnóstico reciente, de 17 o más años de edad, era evaluada por primera vez por el servicio de Infectología, era invitada a recibir el apoyo de parte de PPP. Con la intención de preservar a los integrantes de PPP, se excluyeron de la invitación a quienes, a juicio del equipo de salud, pudieran constituir un riesgo físico o psíquico para los integrantes de PPP (personas violentas y sujetos cuya gravedad clínica hiciera prever una sobrevida corta).

Quienes aceptaban la propuesta firmaban un consentimiento y a partir de concretar el primer encuentro con un integrante de PPP pasaban a formar parte del grupo intervención. Las PVVIH con diagnóstico reciente que rechazaban la propuesta, quienes no llegaran a recibirla (por mal estado físico o psíquico inicial y falta de oportunidad ulterior, porque el paciente faltó a la cita en la que se haría la invitación, por no hallar un momento adecuado, etc.) y quienes tras seis meses de la oferta no respondían (dicho retraso en la respuesta se interpretó como rechazo), conformaron el grupo control (Figura 1A).

Los principales argumentos por los que algunos pacientes rechazaron la propuesta fueron las dudas sobre el respeto a la confidencialidad, a la preservación de la identidad y rechazar la idea de revelar el diagnóstico de infección por VIH a otras personas. Si tras una breve explicación el profesional de la salud no lograba desactivar ese prejuicio, se dejaba de lado la invitación.

La atención de ambos grupos fue idéntica, a excepción del apoyo de PPP a los integrantes del grupo intervención. Dicha 


\section{Figura 1A. Esquema del estudio}

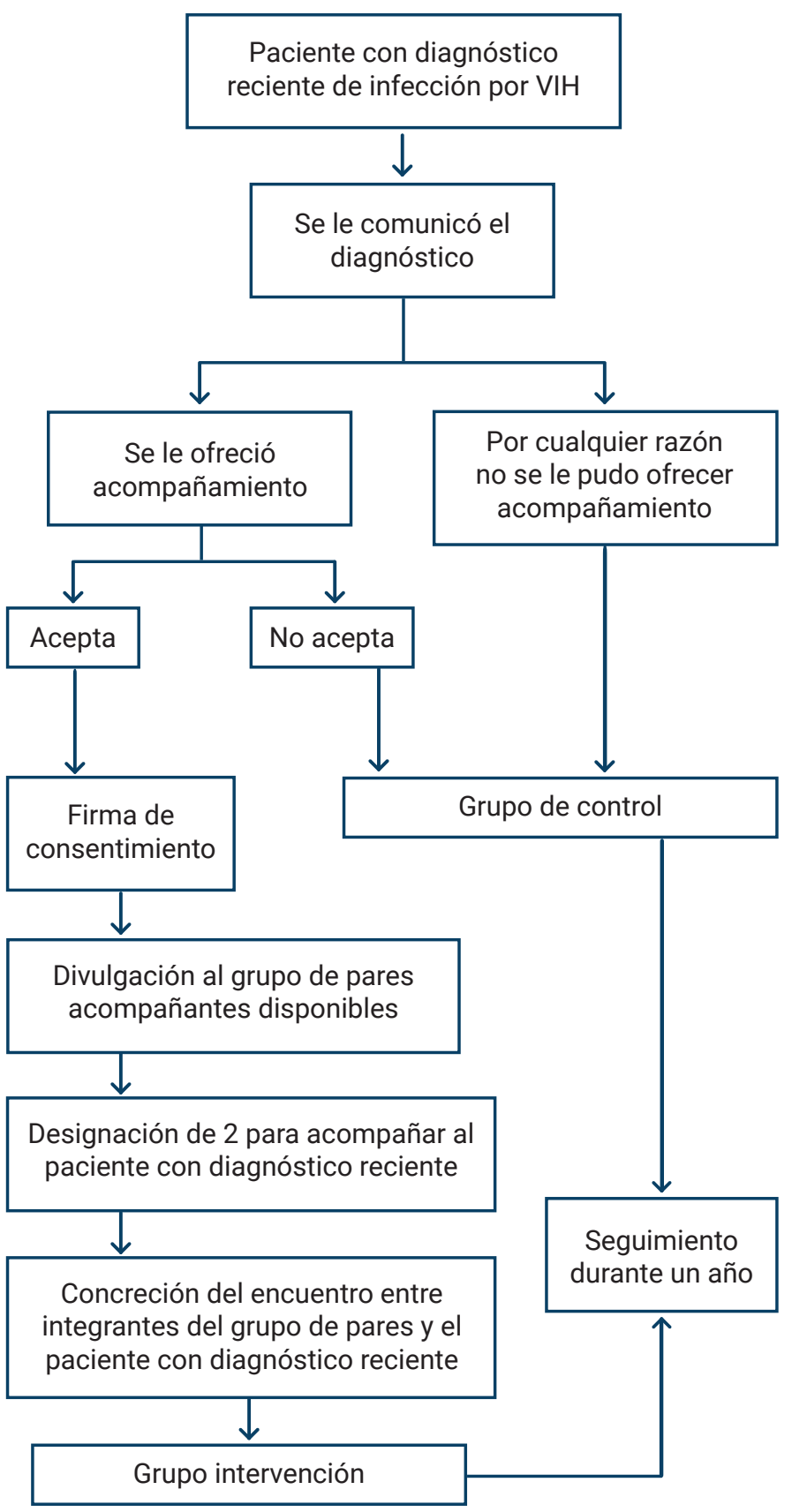

atención siguió la habitualidad de la atención de pacientes del hospital a través del sistema general de otorgamiento de turnos. En el caso de las PVVIH que egresaban de una internación, nuestro servicio los atendía en turnos no programados cada miércoles siguiente al egreso. También se llevaron a cabo citas concretadas informalmente en función de necesidades psicológicas o físicas de los pacientes identificados por algún profesional de la salud.

Laconvocatoriaa los integrantes dePPP para el acompañamiento se hacía mediante correo electrónico o por WhatsApp. Los primeros dos que respondían autopostulándose para el acompañamiento eran designados para el apoyo del nuevo paciente incorporado (Figura 1A).

Al momento de tener su primer encuentro con un integrante de PPP pasaban a integrar el grupo intervención.

Si bien el primer encuentro entre ellos debía ser personal, la forma en que se llevaba adelante el acompañamiento y los ulteriores encuentros fue dejado a su criterio. Se admitió que fuera presencial, telefónico o virtual a través de redes sociales. El contenido del apoyo de los integrantes de PPP era variado: compartir experiencias y vivencias propias acerca del evento de ser una PVVIH que pudieran ayudar a transitar el momento alrededor del diagnóstico, facilitar el acceso a servicios del hospital, facilitar la comunicación con el equipo de salud, resolver dudas sobre la dinámica hospitalaria, sobre la toma de los fármacos o sus efectos adversos hasta que el paciente pueda contactar al médico.

Los integrantes del equipo de salud solo podían influir facilitando las instalaciones del hospital para los encuentros o poniendo en contacto a las partes proveyendo los contactos telefónicos. No hubo remuneración para los integrantes de PPP ni hubo apoyo económico para la realización del estudio. Los viáticos y los gastos para solventar las comunicaciones telefónicas corrieron por cuenta de los integrantes de PPP, sin apoyo externo.

El apoyo de PPP al grupo intervención fue periódicamente supervisado por el equipo de salud mediante encuentros personales o grupales.

\section{Variables analizadas:}

- Proporción de pacientes con buen control clínico: definido como haber cumplido con tres o más visitas médicas durante los 12 meses de estudio.

- La tasa de retiro de TARGA de la farmacia (dato obtenido de los registros de la farmacia y de la plataforma SISA), calculado como el número de meses durante los cuales la PVVIH retiró su provisión mensual de TARGA x 100/ número de meses durante los cuales la indicación de TARGA permaneció vigente. Homologando la tasa de retiro de TARGA a la adherencia y dado que la literatura propone distintos umbrales para definir la correcta adherencia, se evaluó la tasa de retiro de TARGA con tres umbrales, $>80 \%$, $>90 \%$ y $>95 \%$ (en la tabla solo incluimos los resultados de este último umbral). 
- La proporción de pacientes que suspendieron en forma no estructurada el TARGA. La suspensión no estructurada se definió como un período de tres o más meses consecutivos durante los cuales las PVVIH no retiró de la farmacia la provisión de antirretrovirales. Elegimos el periodo de tres meses dado que durante entrevistas a pacientes no adherentes descubrimos que algunos lograban cubrir períodos menores con TARGA proveniente de fuentes no convencionales, como familiares y amigos.

- La proporción de pacientes con buen control de laboratorio, definido como haber concurrido para tres extracciones de sangre para CV y R-CD4 durante el periodo de seguimiento (un número de extracciones que juzgamos necesario y suficiente para evaluar adecuadamente la seguridad y eficacia del TARGA durante el primer año de tratamiento). Dada la exigua cantidad de pacientes que alcanzaron ese objetivo también se determinó la proporción de pacientes que concurrieron para al menos dos extracciones.

- La tasa de las PVVIH que lograron $\mathrm{CV}<50$ copias ARN-VIH/ $\mathrm{mL}$ al menos una vez durante el primer año de seguimiento.

- La proporción de PVVIH que lograban aumentar al menos 50 CD4 durante el primer año de seguimiento.

- La tasa de abandono de seguimiento: definido como la falta de contacto con el sistema de salud por seis meses consecutivos.
Las variables continuas fueron convertidas en variables categóricas y se analizaron como tales (junto con las otras variables categóricas) mediante tablas de contingencia de $2 \times 2$ aplicando la prueba exacta de Fisher. El software utilizado fue Epilnfo 7.2.2.6.

\section{Resultados}

Entre junio de 2014 y agosto de 2018, 227 personas recibieron diagnóstico de infección por VIH.

Al momento de la escritura de este manuscrito, $39 \mathrm{PVVIH}$ llevaban un seguimiento menor a 12 meses desde el primer encuentro con un integrante de PPP, por lo cual no fueron incluidas en este informe. Veintitrés pacientes no fueron incluidos pues nunca concurrieron al consultorio externo (9 casos por continuar su seguimiento en otro centro asistencial; 11 porque fallecieron durante la internación, antes de ser informados del diagnóstico de VIH positivo; 3 se perdieron del seguimiento tras el alta de la internación). Siete pacientes no fueron incluidos porque el equipo de salud, priorizando la salud física y emocional de los integrantes de PPP, juzgó inconveniente ofrecerles el ingreso al programa (en 6 casos por tratarse de personas con muy mal estado de salud que hacía prever una sobrevida corta y en 1 caso por tratarse de una persona con conductas).

La población estudiada quedó conformada por aquellas PVVIH con diagnóstico reciente de infección por VIH que

\section{Tabla 1. Características de la población de PVVIH con diagnóstico reciente incluida en el estudio}

\begin{tabular}{|c|c|c|}
\hline & $\begin{array}{l}\text { Grupo intervención } \\
\qquad \mathrm{n}=\mathbf{4 0}\end{array}$ & $\begin{array}{c}\text { Grupo control } \\
n=118\end{array}$ \\
\hline Género fem / masc / trans & $18 / 21 / 1$ & $48 / 70 / 0$ \\
\hline Edad; mediana (RIC) & $38,4(27,9-50,9)$ & $35,5(26,8-44)$ \\
\hline Procedencia CABA / Gran Buenos Aires / otro & $18 / 22 / 0$ & $62 / 55 / 1$ \\
\hline Nacionalidad argentina; n (\%) & $28(70)$ & $85(72)$ \\
\hline Diagnóstico durante una internación; n (\%) & $11(27,5)$ & $20(16,9)$ \\
\hline Vía adquisición VIH het/homo-bi/otro; n/n/n & $27 / 13 / 0$ & $93 / 23 / 1^{\star}$ \\
\hline Escolaridad máxima alcanzada menor que primaria completa; n (\%) & $4(10)$ & $8(6,9)^{\star \star}$ \\
\hline Desempleados; n (\%) & $15(37,5)$ & $44(37,3)$ \\
\hline Usuario de drogas o consumo excesivo de alcohol; n (\%) & $14(35)$ & $49(42,6)^{\star \star \star}$ \\
\hline CDC B o C; n (\%) & $12(30)$ & $27(22,9)$ \\
\hline R-CD4 basal <200 células/mm³; n (\%) & $22(55)$ & $45(39,1)^{\star \star \star}$ \\
\hline Iniciaron TARGA; n (\%) & $40(100)$ & $97(82,2)$ \\
\hline
\end{tabular}

*Un paciente con vía de adquisición desconocida

**En 2 casos se ignoraba el dato

***En 3 casos se ignoraba el dato 
concretaron al menos una visita al consultorio externo de infectología del HGADFS (n=158). El grupo intervención quedó integrado por $40 \mathrm{PVVIH}$ que tuvieron al menos un encuentro con un integrante de PPP. Los 118 restantes integraron el grupo control. Los integrantes del grupo intervención presentaron enfermedad más avanzada, una menor mediana de R-CD4 y una mayor proporción de internaciones. Por lo demás eran similares (Tabla 1).
EI TARGA fue iniciado por $100 \%$ de los integrantes del grupo intervención y $87,3 \%$ de los integrantes del grupo control [RR 1,15 (IC95 1,07-1,23); p=0,024] (dato no mostrado en la tabla).

La mortalidad fue igual en ambos grupos: 1 paciente del grupo intervención y 3 del grupo control fallecieron antes de los tres meses de seguimiento [2,5\% vs 2,54\%; RR 0,98\% (IC95 0,11$9,19) \mathrm{p}=1$ ] (dato no mostrado en las tablas). Tras excluir a los

\section{Tabla 2. Control clínico, de laboratorio y seguimiento de las PVVIH con diagnóstico reciente tras excluir derivados y fallecidos}

\begin{tabular}{|l|c|c|c|c|} 
& $\begin{array}{c}\text { Grupo intervención } \\
\mathbf{n = 3 7}\end{array}$ & $\begin{array}{c}\text { Grupo control } \\
\mathbf{n = 1 1 2}\end{array}$ & RR (IC 95) \\
\hline Pacientes con buen control clínico; $\mathrm{n} / \mathrm{n}(\%)$ & $35 / 37(94,6)$ & $85 / 112(75,9)$ & $4,2(1,08-16,6)$ & 0,015 \\
\hline Pacientes con >1 extracción de sangre; $\mathrm{n} / \mathrm{n}(\%)$ & $21 / 37(56,8)$ & $36 / 112(32,14)$ & $2,1(2,21-3,71)$ & 0,013 \\
\hline Pacientes con 3 extracciones de sangre; $\mathrm{n} / \mathrm{n}(\%)$ & $4 / 37(10,8)$ & $11 / 112(9,8)$ & $1,08(0,44-2,64)$ & 1 \\
\hline Pacientes con abandono de seguimiento; $\mathrm{n} / \mathrm{n}(\%)$ & $3 / 37(8,1)$ & $29 / 112(25,9)$ & $0,3(0,11-0,98)$ & 0,02
\end{tabular}

\section{Figura 1B. Esquema del estudio}

227 pacientes con diagnóstico de infección por VIH

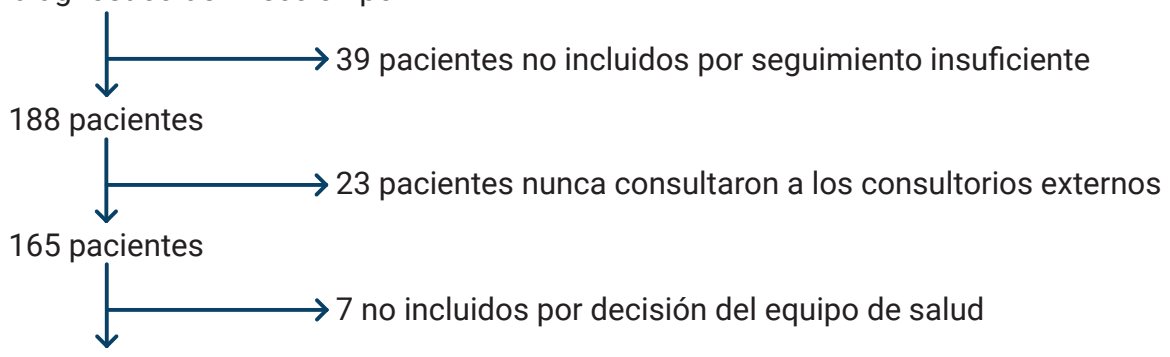

158 pacientes incluidos en la evaluación

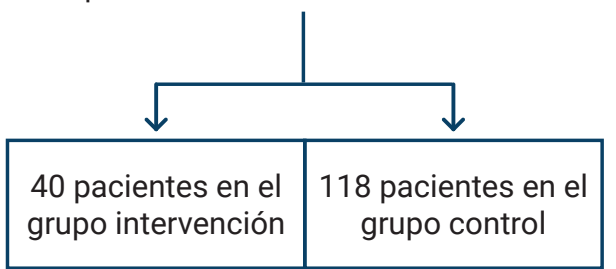

PVVIH con al menos 1 visita al

consultorio externo de Infectologia

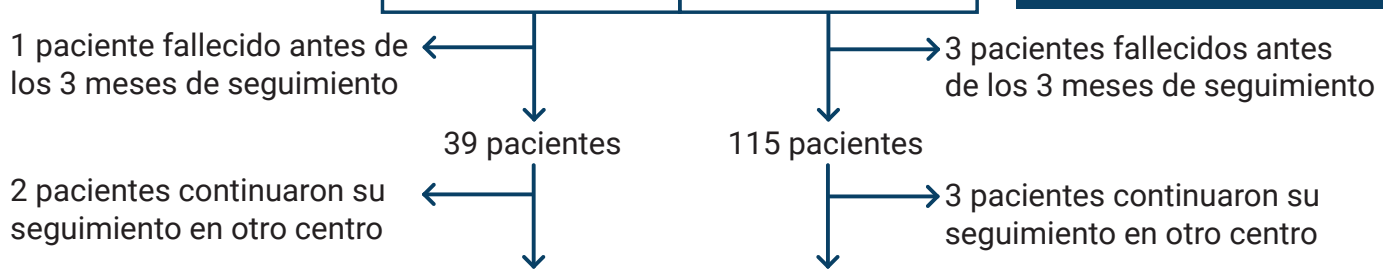

2 pacientes continuaron su

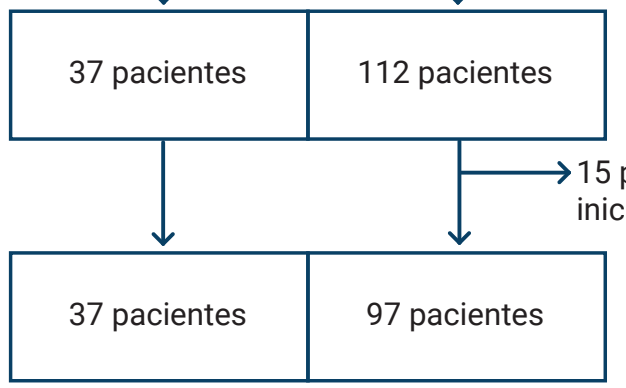

PVVIH con con al menos 1 visita al consultorio externo de Infectologia y 12 meses de seguimiento

15 pacientes nunca iciaron TARGA

PVVIH con con al menos 1 visita al consultorio externo de Infectologia, 12 meses de seguimiento y consumo de TARGA 
fallecidosy a los que continuaron su seguimiento en otros centros asistenciales ( 2 pacientes del grupo intervención y 3 del grupo control), quedaron $149 \mathrm{PVVIH}$ (37 en el grupo intervención y 112 en el grupo control) que completaron 12 meses de seguimiento en el servicio de infectología. En el grupo intervención se observó un mejor control clínico, mayor número de extracciones de sangre para determinación de CV y R-CD4 y menor abandono de seguimiento (Tabla 2).

Para analizar los resultados en quienes iniciaron TARGA, se excluyeron a los 15 pacientes que no lo iniciaron. Entre quienes no iniciaron TARGA no identificamos ninguna característica que los convirtiera en un subgrupo particularmente vulnerable. Las PVVIH que iniciaron TARGA y continuaron su atención en nuestro hospital fueron 134 (37 en el grupo intervención y 97 en el grupo control) (Figura 1B). En el grupo intervención se observó un mayor porcentaje de pacientes con adecuado retiro de TARGA de la farmacia; una menor tasa de suspensiones no estructuradas del tratamiento y una mayor proporción de pacientes que alcanzaron CV indetectable. La recuperación inmunológica fue similar entre ambos grupos (Tabla 3).

\section{Discusión}

En esta experiencia, la implementación de un programa de acompañamiento para $\mathrm{PVVIH}$ con diagnóstico reciente de infección por VIH por parte de otras PVVIH motivadas y entrenadas (a quienes denominamos pares acompañantes) mostró utilidad para alcanzar mejores resultados en varios indicadores de adherencia, comparado con lo observado entre quienes no recibieron el apoyo de pares.

Las experiencias publicadas muestran diferentes grados de eficacia sobre distintos parámetros en distintos aspectosyáreas de la salud de las PVVIH.
La principal fortaleza de nuestro estudio consiste en la elección de la población sobre la cual se decidió trabajar: PVVIH que recibieron recientemente su diagnóstico de infección por $\mathrm{VIH}$, un momento de particular labilidad y vulnerabilidad en su historia personal (37). Hay pocas publicaciones que hayan focalizado en esa población $(38,40)$.

La incorporación de pares al cuidado de PVVIH con diagnóstico reciente podría ayudar a un mejor posicionamiento inicial frente a la infección, contribuir a la resolución de dudas, desmitificar aspectos de la infección por VIH y del TARGA, colaborar en derribar imaginarios negativos como el de la muerte inminente y la imposibilidad de tener descendencia, orientar a la persona involucrada dentro de los centros de salud y contribuir a su empoderamiento para mejorar sus posibilidades de adquirir las herramientas necesarias para continuar con su atención médica, permanecer incluida en el sistema de salud, logar una mejor adherencia al TARGA y mejores resultados de los parámetros clínicos y de laboratorio.

Sostenemos la hipótesis de que el trato directo entre una persona con reciente diagnóstico de infección por VIH y otra que ya ha transitado esa instancia y asumido su diagnóstico, ofrecería una oportunidad de identificación, un interlocutor válido para resolver dudas personales en forma directa, a través de la construcción de una relación cercana, no necesariamente duradera, pero eficaz en el contexto de la coyuntura. La contención ofrecida por los pares a las PVVIH con diagnóstico reciente de infección por VIH podría actuar como un elemento preventivo o moderador de la eventual depresión que frecuentemente acontece tras el diagnóstico de infección por VIH $(41,42)$. Un par podría ser, en el contexto del diagnóstico reciente, un confidente con el cual poder compartir la noticia del diagnóstico, lo cual alivia y prepara para una mejor adherencia futura, como lo sugieren algunas publicaciones (43).

\section{Tabla 3. Parámetros de analítica sanguínea y uso de TARGA de las PVVIH con diagnóstico} reciente tras excluir a quienes no iniciaron TARGA

\begin{tabular}{|c|c|c|c|c|}
\hline & $\begin{array}{l}\text { Grupo intervención } \\
\qquad \mathrm{n}=\mathbf{3 7}\end{array}$ & $\begin{array}{l}\text { Grupo control } \\
\mathrm{n}=97\end{array}$ & RR (IC 95) & $\mathbf{P}$ \\
\hline Tasa de retiro de TARGA de farmacia >95\%; n/n (\%) & $19 / 37(51,4)$ & $18 / 97(18,6)$ & $2,77(1,64-4,66)$ & 0,0003 \\
\hline $\begin{array}{l}\text { Pacientes con suspensión no estructurada del } \\
\text { TARGA; } n / n(\%)\end{array}$ & $4 / 37(10,8)$ & $35 / 97(36,1)$ & $0,30(0,11-0,78)$ & 0,008 \\
\hline $\begin{array}{l}\text { Pacientes con TARGA que lograron } \mathrm{CV}<50 \\
\text { copias } \mathrm{VIH} / \mathrm{mL} ; \mathrm{n} / \mathrm{n}(\%)^{\star}\end{array}$ & $31 / 31(100)$ & $57 / 67(85,1)$ & $1,18(1,06-1,30)$ & 0,06 \\
\hline $\begin{array}{l}\text { Pacientes bajo TARGA que lograron aumento }>50 \\
\mathrm{CD} 4 / \mathrm{mm} 3 ; \mathrm{n} / \mathrm{n}(\%) *\end{array}$ & $22 / 30(73,3)$ & $47 / 64(73,4)$ & $0,99(0,51-1,94)$ & 1 \\
\hline
\end{tabular}

*Solo se consideraron a quienes hicieron al menos una extracción para análisis de CV y/o R-CD4. Para definir el aumento del CD4 se utilizó el último valor disponible de dicha determinación 
El estudio presenta limitaciones. La muestra evaluada es pequeña, no solo porque se incluyeron solo $158 \mathrm{PVVIH}$ con diagnóstico reciente, sino también por la diferencia de magnitud entre el grupo intervención y el grupo control (40 vs 118). Esto fue el resultado de una alta tasa de no-aceptación a la propuesta de acompañamiento.

La distribución de los pacientes en los grupos estudiados no fue aleatorizada. El grupo intervención quedó conformado por quienes aceptaron la propuesta, lo cual le dio homogeneidad a la muestra; pero el grupo control quedó integrado por personas que rechazaron la propuesta, no la recibieron (por distintas razones) o no respondieron tras seis meses de haber recibido la oferta (lo que fue interpretado como rechazo), es decir que los motivos para no ingresar al grupo intervención fueron heterogéneos. Nos preguntamos: ¿habrían aceptado la propuesta quienes nunca la recibieron? ¿por qué motivo algunos no respondieron luego de seis meses de haber recibido la propuesta? De haber aceptado ¿habrían tenido una mejor evolución que la observada? La peor evolución observada en el grupo control ¿fue resultado de la falta de contacto con PPP? ¿o se trató de individuos con alguna característica basal -no identificada por este estudio- que hacía prever una mala evolución que no sería revertida por la intervención de pares? Asumimos como una limitación del estudio la conformación de un grupo control con esa heterogeneidad.

Como resultado de entrevistas informales a los integrantes del grupo control, descubrimos que los principales motivos del rechazo fueron la duda sobre el respeto a la confidencialidad y la negativa a revelar el diagnóstico a terceros, argumentos que permiten suponer que existe temor a la estigmatización. Ello revela que tanto la sociedad como el sistema de salud siguen en deuda al no resolver uno de los principales componentes del imaginario de la población: el estigma.

En relación con lo anterior, y en función de experiencias publicadas $(44,45)$, nos preguntamos si los malos resultados observados en el grupo control son consecuencia de la falta de apoyo de PPP o de que se trató de un grupo más sensible a percibir una eventual estigmatización, lo cual lleva a aislamiento social y depresión. No evaluamos variables que pudieran contestar estas preguntas, ya que no formó parte de la hipótesis inicial y la respuesta queda pendiente para futuras investigaciones.

El programa de apoyo de pares de nuestro hospital, concebido como lo fue, es una iniciativa cuya sustentabilidad en el tiempo es incierta, ya que no logramos conseguir apoyo económico ni técnico. La tarea de PPP fue sostenida gracias a la dedicación y compromiso de las PVVIH asistidas en el consultorio externo de Infectología durante muchos años, quienes, al recibir la propuesta de formar parte de PPP, aceptaron gustosos y ratificaron el entusiasmo inicial en cada encuentro con quienes recibían su diagnóstico. El sostén económico imprescindible para realizar traslados o comunicaciones telefónicas fue absorbido por los integrantes de PPP. Otros equipos de la región informan situaciones similares (35).

Todo esto, junto al carácter local de la experiencia, limitan la generalización en la aplicabilidad de los resultados. Sin embargo, y siendo conscientes de las limitaciones, esos mismos resultados nos entusiasman y nos permiten mantener la intención de involucrar a más PVVIH para apoyo de sus pares, pues creemos que el acompañamiento por parte de "quien ya estuvo alli” podría crear una corriente de confianza y credibilidad mayor que la que generan las propias instituciones y los propios profesionales de la salud. Por último, el testimonio de los integrantes de PPP afirmando que el acompañamiento constituye una oportunidad para el desarrollo personal (un resultado ciertamente no buscado pero que produjo una grata sorpresa en el equipo), algo ya referido en la literatura (45), ratifica que el camino es el correcto.

En general, la baja cantidad de pacientes incluidos en las publicaciones, los cortos periodos de seguimiento, las diferentes estrategias aplicadas, las diferencias culturales y geográficas de los sitios en los cuales se implementaron y los variados resultados obtenidos, no permiten aún definir si la estrategia de acompañamiento de pares resultará beneficiosa para mejorar la adherencia y los resultados de laboratorio y clínicos con respecto a las estrategias estándares como para marcar una diferencia en la evolución de las PVVIH que reciben TARGA. Tampoco queda clara la durabilidad de los eventuales resultados positivos, ya que distintos estudios, con diferente tiempo de seguimiento, han arrojado resultados diferentes e incluso contradictorios. La experiencia aquí presentada nos permite proponer que, a la hora de decidir la incorporación de pares al cuidado de las PVVIH, cada sitio deberá diseñar la propia modalidad y definir la población blanco sobre la cual trabajar. Consideramos que este es un estudio piloto, un punto de partida para seguir investigando la estrategia.

\section{Agradecimientos}

Al Dr. Daniel Lerman, por los permisos otorgados a la licenciada Mónica Pasarón; al centro de jubilados María Ana Mogas, de la plaza Madre María Ana Mogas-Sector Parque Santojanni, que generosamente nos facilitó sus instalaciones para poder realizar allí nuestros encuentros; 
al Dr. Pablo Copertari, por su paciencia y ayuda.

Y, fundamentalmente, a Sergio, Hugo, Olguita, Graciela, Andrea, Gaby, Roxana, Carlos, Ale, Blanca y Viviana, los verdaderos protagonistas de esta experiencia, nuestros queridos PPP. Autodidactas en un camino riguroso y ríspido, y que hoy, habiendo aprendido la técnica de la caminata, deciden, heroicamente, desde el pavimento, colaborar amorosamente con quienes recién lo inician. $Y$ siguen aprendiendo a seguir enseñando. Nuestro agradecimiento y admiración. 


\section{Referencias}

1. Lima VD, Hoggs RS, Harrigan PR, Moore D, Yip B, Wood $\mathrm{E}$, et al. Continued improvement in survival among HIV-infected individuals with newer forms of highly active antiretroviral therapy. AIDS 2007; 21: 685-92.

2. Lewden C, Chene G, Morlat P, Raffi F, Dupon M, Dellamonica $P$, et al. HIV-infected adults with a CD4 cell greater than 500 cell/mm3 on long-term combination antiretroviral therapy reach same mortality rates as the general population. J Acquir Immune Defic Syndr 2007; 46: 72-7.

3. Panel on treatment of HIV-infected pregnant women and prevention of perinatal transmission. Recommendations for use of antiretroviral drugs in pregnant HIV-1-infected women for maternal health and interventions to reduce perinatal HIV transmission in the United States. Disponible en http://aidsinfo. nih.gov/contentfiles/lvguidelines/PerinatalGL.pdf Acceso el 15 de julio de 2018.

4. Donnell D, Baeten JM, Kiarie J, Thomas KK, Stevens W, Cohen CR, et al. Heterosexual HIV-1 transmission after initiation of antiretroviral therapy: a prospective cohort analysis. Lancet 2010; 375: 2092-8.

5. Loutfy MR, Wu W, Letchumanan M, Bondy L, Antoniou $T$, Margolese $S$, et al. Systematic review of HIV transmission between heterosexual serodiscordant couples where the HIV positive partner is fully suppressed on antiretroviral therapy. PLOS ONE 2013; 8: e55747.

6. Paterson DL, Swindells S, Moht J, Brester M, Vergis EN, Squier C, et al. Adherence to protease inhibitor therapy and outcomes in patients with HIV infection. Ann Intern Med 2000; 133: 21-30.

7. Li JZ, Gallien S, Ribaudo H, Heisey A, Bangsberg DR, Kuritzkes DR. Incomplete adherence to antiretroviral therapy is associated with higher levels of residual HIV-1 viremia. AIDS 2014; 28: 181-6.

8. Von Wyl V, Klimkait T, Yerly S, Nicca D, Furrer $H$, Cavassini $\mathrm{M}$, et al, and the Swiss HIV Cohort Study. Adherence as a predictor of the development of class specific resistance mutations: The Swiss HIV Cohort Study. PLOSS ONE 2013; 8: e77691.

9. Wood E, Hogg RS, Yip B, Harrigan PR, O'Shaughnessy MV, Montaner JS. Effect of medication adherence on survival of HIV-infected adults who start highly active antiretroviral therapy when the CD4+ cell count is 0.200 to $0.350 \times 10$ (9) cells/L. Ann Intern Med 2003; 139: 810-6.

10. Booth CL, Geretty AM. Prevalence and determinants of transmitted antiretroviral drug resistance in HIV-1 infection. J Antimicrob Chemother 2007; 59: 1047-56.

11. Sabaté E. Organización Mundial de la Salud/OMS 2004. Adherencia a los tratamientos a largo plazo. Pruebas para la acción. Disponible en www.paho. org/Spanish/ad/dpc/nc/adherencia-largo-plazo.pdf. Acceso el 15 de julio de 2018.

12. Lazo M, Gange SJ, Wilson TE, Anastos K, Ostrow DG, Witt MD, et al. Patterns and predictors of changes in adherence to highly active antiretroviral therapy: longitudinal study of men and women. Clin Infect Dis 2007; 45: 1377-85.

13. Mbonye M, Seeley J, Ssembajja F, Birungi J, Jaffar S. Adherence to antiretroviral therapy in Jinja, Uganda: a six-year follow-up study. PLOS ONE 2013; 8: e78243

14. CHECCOS A. C. Comité Humanitario de Esfuerzo Compartido contra el SIDA A.C. Manual de Asesoría y Acompañamiento de Pares. As-Par: Asesoría y Acompañamiento de Pares para la incorporación y utilización de los servicios de salud por personas con VIH. http://checcos.org.mx/wp-content/ uploads/2013/12/AS-PAR.-Asesor\%C3\%ADa-yAcompa\%C3\%B1amiento-de-Pares.pdf. Acceso el 15 de julio de 2018.

15. ONUSIDA. Educación interpares y VIH/SIDA: Conceptos, usos y problemas. http://data.unaids. org/publications/irc-pub01/jc291-peereduc_es.pdf. 2000. Acceso el 15 de julio de 2018.

16. Liu $\mathrm{Y}$, Vermund $\mathrm{SH}$, Ruan $\mathrm{Y}$, Liu H, Rivet Amico K, Simoni JM. Peer counseling versus standard-ofcare on reducing high risk behaviours among newly diagnosed HIV-positive men who hav sex with men in Beijing, China: a randomized intervention study. J Int AIDS Soc 2018; 21. 0:e25079.

17. Konate I, Traore L, Ouedraogo A, Sanon A, Diallo R, Ouedraogo JL, et al. Linking HIV prevention and care for community interventions among high-risk women in Burkina Faso--the ARNS 1222 "Yerelon" cohort. J Acquir Immune Defic Syndr 2011; 57 Suppl 1: S50-4.

18. 1Mburu G, Ram M, Skovdal M, Bitira D, Hodgson I, Mwai GM, et al. Resisting and challenging stigma in Uganda: the role of support groups of people living with HIV. J int AIDS Soc 2013; 16 (suppl 2): 18636.

19. 1Steward WT, Sumitani J, Moran ME, Ratlhagana MJ, Morris JL, Isidoro L. Engaging HIV-positive clients in care: acceptability and mechanisms of action of a peer navigation program in South Africa. AIDS Care 2018; 30: 330-7.

20. Mahalakshmy T, Premarajan KC, Abdoul H. Correlates of human immunodeficiency virus (HIV) related 
knowledge among HIV infected people. Indian J Dermatol Venereol Leprol 2011; 77: 37-41.

21. Ruria EC, Masaba R, Kose J, Woelk G, Mwangi E, Matu $L$, et al. Optimizing linkage to care and initiation and retention on treatment of adolescents with newly diagnosed HIV infection. AIDS 2017; 31 (suppl 3): S253-60.

22. Enriquez $M$, Cheng AL, Banderas J, Farnan R, Chertoff $K$, Hayes $D$, et al. A Peer-led HIV medication adherence intervention targeting adults linked to medical care but without a suppressed viral load. J Int Assoc Provid AIDS Care 2015; 14: 441-8.

23. Kiweewa FM, Wabwire D, Nakibuuka J, Mubiru M, Bagenda D, Musoke $P$, et al. Noninferiority of a taskshifting HIV care and treatment model using peer counselors and nurses among Ugandan women initiated on ART: evidence from a randomized trial. J Acquir Immune Defic Syndr 2013; 63: e125-32.

24. Simoni JM, Nelson KM, Franks JC, Yard SS, Lehavot K. Are peer interventions for HIV efficacious? A systematic review. AIDS Behav 2011; 15: 1589-95.

25. Abdulrahman SA, Rampal L, Ibrahim F, Radhakrishnan AP, Kadir Shahar $H$, Othman N. Mobile phone reminders and peer counseling improve adherence and treatment outcomes of patients on ART in Malaysia: A randomized clinical trial. PLoS One 2017; 6; 12: e0177698.

26. Chang LW, Kagaayi J, Nakigozi G, Ssempijja V, Packer $\mathrm{AH}$, Serwadda D, et al. Effect of peer health workers on AIDS care in Rakai, Uganda: a cluster-randomized trial. PLOS ONE 2010; 5: e10923.

27. Pearson CR, Micek MA, Simoni JM, Hoff PD, Matediana E, Martin DP, et al. Randomized control trial of peerdelivered, modified directly observed therapy for HAART in Mozambique. J Acquir Immune Defic Syndr 2007; 46: 238-44.

28. Simoni JM, Huh D, Frick PA, Pearson CR, Andrasik MP, Dunbar PJ, et al. Peer support and pager messaging to promote antiretroviral modifying therapy in Seattle: a randomized controlled trial. J Acquir Immune Defic Syndr 2009; 52: 465-73.

29. Ruiz I, Olry A, López MA, Prada JL, Causse M. Prospective, randomized, two-arm controlled study to evaluate two interventions to improve adherence to antiretroviral therapy in Spain. Enferm Infecc Microbiol Clin 2010; 28: 409-15.

30. Cuong DD, Sönnerborg A, Van Tam V, El-Khatib Z, Santacatterina M, Marrone G, et al. Impact of peer support on virologic failure in HIV-infected patients on antiretroviral therapy - a cluster randomized controlled trial in Vietnam. BMC Infect Dis 2016; 16: 759.

31. Williams $A B$, Wang $H$, Li $X$, Chen J, LI L, Fennie K. Efficacy of an evidence-based ARV adherence intervention in China. AIDS Patient Care STDS 2014; 28: 411-7.

32. Kanters S, Park JJH, Chan K, Ford N, Forrest J, Thorlund $\mathrm{K}$, et al. Use of peers to improve adherence to antirretroviral therapy: a global network metaanalysis. J Int AIDS Soc 2016; 19: 21141.

33. Picelli I, Díaz-Bermúdez XP. Will these drugs be worthwhile? An anthropological study of adherence to antiretroviral therapies among support groups for people living with HIV and AIDS. Saude Soc 2014; 23: 44-57.

34. Campero L, Caballero M, Kendall T, Herrera C, Zarco A. Soporte emocional y vivencias del VIH: Impactos en varones y mujeres mexicanos desde un enfoque de género. Salud Mental 2010; 33: 409-17.

35. Arístegui I, Dorigo A, Bofill L, Bordatto A, Lucas $M$, Fernandez Cabanillas $G$, et al. Obstáculos a la adherencia y retención en los sistemas de salud público y privado según pacientes y personal de salud. Actual SIDA Infectol 2014; 22: 71-80.

36. Warley E, Salas M, Vieni MI, Szyld E. Adherencia a las terapias antirretrovirales y al cuidado de la salud: experiencia de un programa de apoyo entre pares. Actualizaciones en sida 2011; 19: 40-5.

37. Atkinson JH, Higgins JA, Vigil O, Dubrow R, Remien $\mathrm{RH}$, Steward WT, et al. Psychiatric context of acute/ early HIV infections. The NIMH multisite acute HIV infection study: IV. AIDS Behav 2009; 13:1061-7.

38. Pecheny $M$ (director del proyecto), Binstock $G$, Manzelli H, Hiller R, Bruno M (equipo de investigación) Caracterización de las mujeres recientemente diagnosticadas con VIH en Argentina. Informe final 2012. http://www.msal.gob.ar/sida/images/ stories/4-publicaciones/pdf/2014-11_informe-finalmujeres-VIH.pdf. Acceso 2 de mayo de 2018.

39. Moskowitz JT, Wrubel J, Hult JR, Maurer S, Acree M. Illness appraisals and depression in the first year after HIV diagnosis. PLOS ONE 2013; 8: e78904.

40. Prestage G, Brown G, Allan B, Ellard J, Down I. Impact of peer support on behavior change among newly diagnosed australian gay men. J Acquir Immune Defic Syndr 2016; 72: 565-71.

41. Bhatia R, Hartman C, Kallen MA, Graham J, Giordano TP. Persons newly diagnosed with HIV infection are at high risk for depression and poor linkage to care: results from the Steps Study. AIDS Behav 2011; 15: 1161-70. 
42. Grav S, Hellzén O, Romild U, Stordal E. Association between social support and depression in the general population: the HUNT study, a cross-sectional survey. J Clin Nurs 2012; 21: 111-20.

43. Nachega JB, Morroni C, Zuniga JM, Sherer R, Beyrer C, Solomon Z, et al. HIV-Related Stigma, Isolation, Discrimination, and Serostatus Disclosure: A Global Survey of 2035 HIV-Infected Adults. Journal of the International Association of Physicians in AIDS Care 2012; 11:172-178.

44. Loutfy $M$, Johnson M, Walmsley S, Samarina A, Vasquez $\mathrm{P}$, Hao-Lan $\mathrm{H}$, et al. The Association Between HIV Disclosure Status and Perceived Barriers to Care Faced by Women Living with HIV in Latin America, China, Central/Eastern Europe, and Western Europe/ Canada. AIDS Patient Care STDs 2016; 30: 435-44.

45. Higa DH, Marks G, Crepaz N, Liau A, Lyles CM. Interventions to improve retention in HIV primary care: a systematic review of U.S. studies. Curr HIV/ AIDS Rep 2012; 9: 313-25. 
Peer support program for people with recently diagnosed HIV-infection: PPP experience

Introduction: To improve retention in the health system of recently diagnosed people living with HIV (PLHIV) and promote their adherence, the "Positive for Positive" peer support program (PPP) was implemented.

Materials and methods: PLHIV with excellent adherence were trained and offered to integrate PPP. Between June/2014 and August/2018 each individual with a recent diagnosis of HIV infection was invited to contact PPP. Patients were prospectively evaluated for one year and variables linked to adherence were analyzed. Their results were compared with those observed among recently diagnosed PLHIV without peer support. It was analyzed using $2 \times 2$ tables and Fisher's exact test (Epilnfo7.2.2.6).

Results: 158 PLHIV were included (40 intervention group and 118 control group). In the intervention group more patients started HAART [100\% vs 87.3\%; RR 1.15 (IC95 1.07-1.23); $\mathrm{p}=0.024]$. After excluding referrals and early deaths, remained 37 and 112 patients, respectively. In the intervention group there was better clinical control [ $94.6 \%$ vs $75.9 \%$; RR 4.2 (IC95 1.08-16.6); $p=0.015]$ and less dropout from follow-up [8.1\% vs 25.9\%; RR 0.3 (IC95 0.11-0.98); p=0.02]. Among those who initiated HAART and had at least one visit to the Infectious Disease Outpatient Clinic (37 intervention group and 97 control group), more patients showed a high refill rate (51.4\%vs. $18.6 \%$ ); RR 2.77 (IC95 1.64-4.66); $p=0.0003]$; greater achievement of undetectable viral load [100\% vs $85.1 \%$; RR 1.18 (IC95 1.061.30); $p=0.06]$; fewer unstructured HAART interruptions [10.8\% vs $36.1 \%$; RR 0.3 (IC95 0.11-0.78); $p=0.008$ ].

Conclusion: Peer support had a positive impact on adherence among recently diagnosed PLHIV in the first year of follow-up.

Keywords: HIV, adherence, peer, recent diagnosis. 